\title{
Motion Control Realization for an Intelligent Car with Integrity Constraints
}

\author{
Yuchuan Li* \\ Department of Automation, Tsinghua University, Beijing 100084, China \\ "Corresponding author
}

\begin{abstract}
In order to realize the obstacle avoidance and tracking movement function of the 3-wheeled intelligent car with integrity constraints, the kinematics model of the car is derived first, and the control of the speed of the car-driven DC motor, i.e. the wheel speed, is realized by using the PWM control method. An improved PID control algorithm and its controller are designed, and some parameters of the controller are regulated according to experimental test data, such as 'hold the ball time' of the car, the turning radius and the turning speed of the car during the obstacle avoidance. Finally, the car can meet the good athletic performances, and achieved good results in the competition.
\end{abstract}

Keywords- Integrity Constraints; Intelligent Car; Improved PID control algorithm; Kinematics model

\section{INTRODUCTION}

Small intelligent cars are important parts of the research field of the wheeled robot. It is also a type of autonomous ground vehicle (AGV). It has wide application prospect in all fields of social life[1-4]. The common smart car has 4 wheels, with one or more motors and servos, as shown in Figure 1(a), the traveling speed of the trolley is realized by controlling the rotational speed of the motor mounted on the rear wheel. It is realized by controlling the steering gear installed in the front wheel position to change direction so that the car with such a structure obviously can not move to its left or right at $90^{\circ}$. And in any case the control, the car cannot realize turning at a radius of 0 . Such a smart car is usually referred to as cars "having non-integrity constraints" [5-6]. In order to achieve a turning at a radius of 0 or a turning at a small radius and wide-angle for a smart car and better meet the needs of industrial and living applications, we need to study small intelligent cars with integrity constraints. In this paper, a 3-wheel-drive intelligent car with integrity constraints is designed, as shown in Figure 1(b). The three wheels of the 3-wheel smart car are driven by three separate motors. By controlling the speeds of the three motors, the car can turn to left or right within a radius of 0 or a turning at wide-angle. Owing to its special structure of the 3-wheel smart car, when designing it to perform some functions, it is necessary to study its equations of motion and corresponding control algorithms.

In this paper, the 3-wheel intelligent car with integrity constraint that can achieve obstacle avoidance and tracking motion is taken as the research object. Based on its kinematic law, the kinematics model of the car is deduced, which is the relationship between car wheel speed and the speed and direction of the car. The control of the speed of wheels is also realized by the PWM control method of the DC motor.
Furthermore, in order to enhance the ability of car movement, an improved PID control algorithm is designed and its controller is realized. In debugging, several parameters of the controller were adjusted in order to have a better motor function.

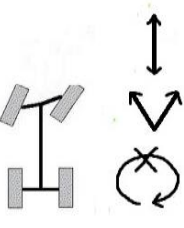

(a)

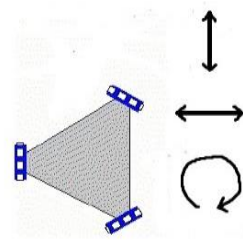

(b)
FIGURE I. A CAR WIT H INTEGRITY CONSTRAINT S AND ANOT HER WITH NON-INTEGRIT Y CONST RAINT S.

\section{THE KINEMATICS MODEL AND MOTION CONTROL ALGORITHM}

\section{A. The Kinematics Model}

The proposed and designed 3-wheel integrity constraints intelligent car is shown in Figure 2. There is a groove in the front of the car which can control the ball in the contest. The axis of the three wheels are in the same plane space and have an angle of $120^{\circ}$ to each other. In addition, the three wheels are independently controlled by three DC motors.

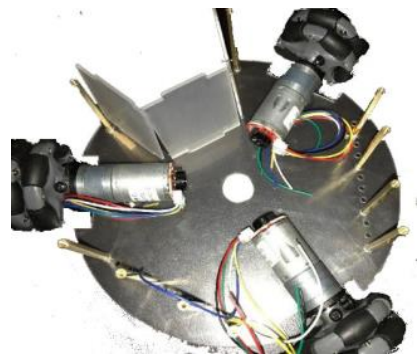

FIGURE II. PICT URE OF WHEELS, BEARINGS AND MOT ORS OF THE CAR.

No matter what kind of complex functions the intelligent car achieve, it must be able to follow our control rules. In this case, the controlled objects are the rotation of three wheels and their speed, that is to build a relationship between the three cons traints of wheels motion and the movement of the center of the car. After that, the car can be controlled by algorithms written in the single-chip microcomputer (SCM). 
In order to get the correspondence relationship between speed of centroid of the car $(\dot{x}$ and $\dot{y})$ and speed of three wheels $\left(V_{1} 、 V_{2}\right.$ and $\left.V_{3}\right)$ or the angular speed of three wheels $\left(\omega_{1}\right.$ 、 $\omega_{2}$ and $\left.\omega_{3}\right)$, establish a coordinate system, as shown in Figure 3.

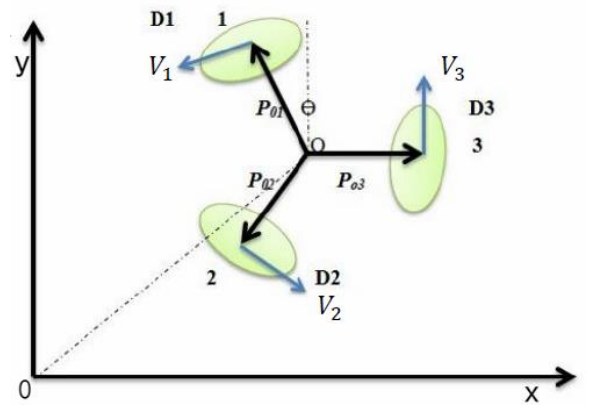

FIGURE III THE COORDINATE SYSTEM OF CAR'S WHEELS AND

In Figure 3, $\theta$ is the angle between the axis of the first wheel and the $y$-axis, which is counterclockwise in Figure 3. The center of mass of the car is $P_{o}(x, y)$, and $l$ is the distance between the wheel center and the centroid of the car. So the coordinates of 3 wheels in the global coordinates can be expressed as compared to the car's center of mass, lead to following equations

$$
P_{o i}=\left[\begin{array}{l}
x_{i} \\
y_{i}
\end{array}\right]=R\left(\theta_{i}\right) \times l\left[\begin{array}{l}
0 \\
1
\end{array}\right], \quad i=1,2,3
$$

$R(\theta)$ is the unitary rotation matrix and it meets the requirements as following equations:

$$
R\left(\theta_{i}\right)=\left[\begin{array}{cc}
\cos \theta_{i} & -\sin \theta_{i} \\
\sin \theta_{i} & \cos \theta_{i}
\end{array}\right]
$$

The direction of the three wheels can be calculated as:

$$
D_{i}=\frac{1}{l} \times R\left(\frac{\pi}{2}\right) \times P_{o i}, i=1,2,3
$$

In the global coordinate system, the position of each wheel is shown in following equations:

$$
P_{i}=P_{o}+R\left(\theta+\frac{2 \pi}{3} \times(i-1)\right) \times P_{o i}, \quad i=1,2,3
$$

After taking a derivative with Equation(3), the velocity of each wheel in the global coordinate system is shown in following equation:

$$
v_{i}=\dot{P}_{o}+\dot{R}\left(\theta+\frac{2 \pi}{3} \times(i-1)\right) \times P_{o i}, i=1,2,3
$$

Thus, the translational speed of each wheel can be calculated as [7]:

$$
V_{i}=v_{i}^{T} \times R\left(\theta+\frac{2 \pi}{3} \times(i-1) \times D_{i}\right), i=1,2,3
$$

Put these equations in a matrix, and it becomes the kinematics model of the car:

$$
\left[\begin{array}{l}
V_{1} \\
V_{2} \\
V_{3}
\end{array}\right]=P(\theta) \times\left[\begin{array}{c}
\dot{x} \\
\dot{y} \\
\dot{\theta}
\end{array}\right]
$$

where $P(\theta)=\left[\begin{array}{ccc}-\cos (\theta) & -\sin (\theta) & l \\ -\cos \left(\frac{\pi}{3}-\theta\right) & -\sin \left(\frac{\pi}{3}-\theta\right) & l \\ -\cos \left(\frac{\pi}{3}+\theta\right) & -\sin \left(\frac{\pi}{3}+\theta\right) & l\end{array}\right]$.

According to the kinematic model in equation (6) and equation (2), if the coordinates of the target point is $\left(x_{d}, x_{d}, \theta_{d}\right)$, the center of mass of the car and the translational speed and direction of the three wheels can be calculated.

Then the displacement vector of the target point is shown in following equation:

$$
\left[\begin{array}{l}
x_{e} \\
y_{e} \\
\theta_{e}
\end{array}\right]=\left[\begin{array}{l}
x \\
y \\
\theta
\end{array}\right]-\left[\begin{array}{l}
x_{d} \\
y_{d} \\
\theta_{d}
\end{array}\right]
$$

Then, the velocity vector can be calculated as:

$$
\left[\begin{array}{c}
\dot{x_{e}} \\
\dot{y}_{e} \\
\dot{\theta_{e}}
\end{array}\right]=\left[\begin{array}{c}
\dot{x} \\
\dot{y} \\
\dot{\theta}
\end{array}\right]=P^{-1}(\theta) \times\left[\begin{array}{l}
V_{1} \\
V_{2} \\
V_{3}
\end{array}\right]
$$

Translational speed of the three wheel is [8]:

$$
\left[\begin{array}{l}
V_{1} \\
V_{2} \\
V_{3}
\end{array}\right]=P(\theta) \times\left(-K_{p} \times\left[\begin{array}{l}
x_{e} \\
y_{e} \\
\theta_{e}
\end{array}\right]-K_{I} \times\left[\begin{array}{l}
\int x_{e} \\
\int y_{e} \\
\int \theta_{e}
\end{array}\right]\right)
$$

where $K_{p}=K_{p}{ }^{T}>0, K_{I}=K_{I}^{T}>0$.

After putting equation(9) in equation(8), the speed vector of the target point is:

$$
\begin{gathered}
{\left[\begin{array}{c}
\dot{x_{e}} \\
\dot{y_{e}} \\
\dot{\theta_{e}}
\end{array}\right]=P^{-1}(\theta) \times P(\theta)\left(-K_{p} \times\left[\begin{array}{l}
x_{e} \\
y_{e} \\
\theta_{e}
\end{array}\right]-K_{I} \times\left[\begin{array}{l}
\int x_{e} \\
\int y_{e} \\
\int \theta_{e}
\end{array}\right]\right)} \\
=-K_{p} \times\left[\begin{array}{l}
x_{e} \\
y_{e} \\
\theta_{e}
\end{array}\right]-K_{I} \times\left[\begin{array}{l}
\int x_{e} \\
\int y_{e} \\
\int \theta_{e}
\end{array}\right]
\end{gathered}
$$

In equation(9), the car's translational speed $V_{1}, V_{2}$ and $V_{3}$ are determined by the motor speed $n$, which is determined by the armature voltage of the motor. According to the pulse width modulation (PWM) Technology, the average value of the armature voltage $U$ of the DC motor is proportional to the product of the duty cycle $\alpha$ of the PWM wave and the DC power supply voltage $U_{\mathrm{s}}[9]$, namely: 


$$
U=\alpha U_{s}
$$

Therefore, the speed of the wheels in the open-loop and closed-loop control[10] is shown in equation(12) and (13) respectively.

$$
\begin{gathered}
{\left[\begin{array}{l}
n_{1} \\
n_{2} \\
n_{3}
\end{array}\right] \times 2 \pi r=\left[\begin{array}{l}
V_{1} \\
V_{2} \\
V_{3}
\end{array}\right]} \\
{\left[\begin{array}{l}
n_{1} \\
n_{2} \\
n_{3}
\end{array}\right] \times 2 \pi r=-\left[\begin{array}{l}
V_{1 c}-V_{1} \\
V_{2 c}-V_{2} \\
V_{3 c}-V_{3}
\end{array}\right]}
\end{gathered}
$$

where $r$ is the wheel radius; $V_{1 \mathrm{c}}, V_{2 \mathrm{c}}$ and $V_{3 \mathrm{c}}$ are the feedback of velocities of the three wheels in the closed-loop control.

Then, according to equation (12), (13), (9) and (10), when setting the speed of motors, the car can move straight to target point under the closed-loop control.

Figure 4 is a schematic diagram of the ideal trajectory and the experimental trajectory of the car along the linear trajectory. When target points are set in a straight line, the deviation between the experimentally track and the desired track is small within a centimeter range, which is similar to a straight line. It indicates that when the car will have a good performance in a straight-line tracing movement underclosed-loop control.

Figure 5 shows the ideal trajectory and the experimental trajectory when the car traverses along the circular trajectory. It is obvious that there is a big deviation between the experimental trajectory and the desired circular trajectory. Furthermore, the small ball in the contest is a ping pong ball and it has a diameter of about $4 \mathrm{~cm}$. Therefore, within centimeter range, the performance of the car's circular tracing cannot meet the requirement of stable "carrying ball" during the race. In order to solve the problem of trajectory correction when the intelligent car tracks in the curvilinear motion, motion control algorithms, such as the PID control algorithm, must be implemented on the car's movement.

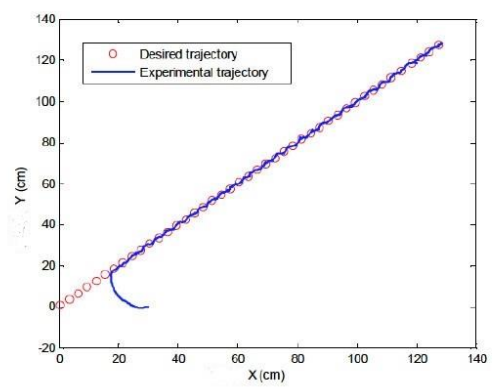

FIGURE IV. THE DESIRED T RAJECTORY AND EXPERIMENT AL TRAJECT ORY OF CAR MOVING STRAIGHT .

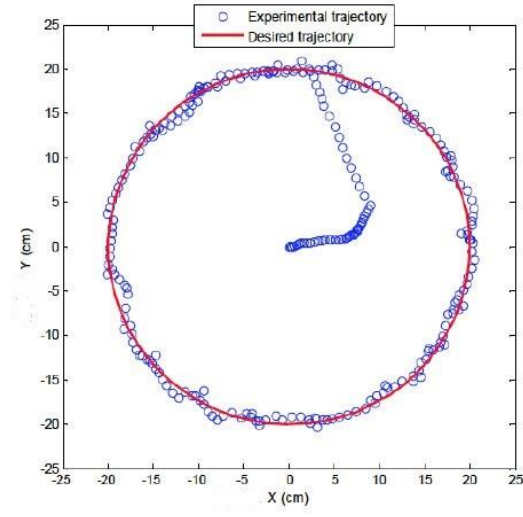

FIGURE V.. THE DESIRED TRAJECTORY AND EXPERIMENT AL TRAJECTORY OF CAR MOVING AS CIRCLE.

\section{B. Motion Control Algorithm}

When the required movement trajectory becomes complicated or the car needs to realize other functions, motion control algorithms are key to stability and reliability of the car's performance. PID controller is the most widely used. It has the advantage of not needing a model, and there are limitations, such as long time for system recovery under impact and strong interference. In order to solve the problem, an improved PID controller is presented.

The ideal PID controller's transfer function can be shown as

$$
\mathrm{D}(\mathrm{s})=K_{p} \times\left(1+\frac{1}{T_{i} s}+T_{d} s\right)=K_{p}+K_{i} \frac{1}{s}+K_{d}
$$

Where $\mathrm{Kp}, \mathrm{Ki}, \mathrm{Kd}$, are factors for the proportion, integral and differential respectively.

In practice, since the time variable $t$ is sampled and it is a discrete value, equation (14) need to be expressed in different forms:

$$
u(k)=u(k-1)+\Delta u(k)
$$

where $\quad u(k-1)=K_{p} e(k-1)+K_{I} \sum_{j=0}^{k-1} e(j)+K_{D}[e(k-$ 1) $-e(k-2)] \quad, \quad$ and $\quad \Delta u(k)=K_{p}(e(k)-e(k-$ 1)) $+K_{I} e(k)+K_{D}[e(\mathrm{k})-2 e(k-1)+e(k-2)]$.

The intelligent car designed in this paper is used in a soccer match as shown in Figure 6. The sources of the control signal are three parts. The first is the judgment signal from the infrared sensor, the second is location signal from the ultrasonic sensor and the third is the "Goal" signal from host venue through bluetooth. 


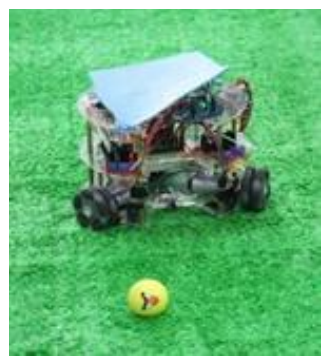

FIGURE VI. PICT URE OF TEST S CAR ON THE SOCCER MATCH FIELD

When the signal from infrared sensor shows that the car is to reach the boundaries, it needs to steer to avoid. When the data collected by multiple ultrasonic sensors processed to become football position information, the car needs to adjust the direction. When the car receives a message "goal" from the host venue, it needs to move back to the original position.

For the first and second part of the signals are collected by the car sensor, there is much high-frequency noise due to the characteristics of the analog signal, as well as othersensors and wire interference. Therefore, the appropriate filter is vital to improve the PID controller.

The differential equation of PID controller can be improved as:

$$
\mathrm{D}_{1}(\mathrm{~s})=K_{p}\left(1+\frac{1}{T_{i} s}+\frac{k \times T_{d} s}{1+\frac{T_{d} s}{K_{d}}}\right)=D_{p i}(s)+D_{d}(s)
$$

And $\Delta u(k)$ in Equation (15) can be calculated, $\Delta u(k)=$ $\Delta u_{p i}(k)+\Delta u_{d}(k)$ :

$$
\Delta u_{p i}(\mathrm{k})=K_{p}(e(k)-e(k-1))+K_{I} e(k)
$$

$$
\begin{gathered}
\Delta u_{d}(k)=\frac{k \times T_{d}}{K_{d} T+T_{d}}\left(\Delta u_{d}(k-1)\right)+K_{d} K_{p}(e(k)-2 e(k-1)+ \\
e(k-2))
\end{gathered}
$$

where $e(k)$ represents unit response.

After low-pass filtering, the integral part of the control equation changes, and $k$ is selected to be 0.6 , then the static error of the car system becomes smaller and the static accuracy improves [11].

\section{TEST S DEBUGGING OF THE CAR}

The automatic control of the car is realized by the state machine algorithm, which means that by setting the transition rules between multiple systemstates, the movement state of the car changes, following the environmental conditions transferred by sensors. Moreover, through the analysis of the kinematic rules and the motion control algorithm, ideal control model can be established. However, there are always some differences between actual conditions and ideal conditions. the following part of the paper shows some of the problems encountered in tests.
The first problem is that it is difficult for the car to keep the speed of the ball at a constant value during "carrying the ball". because the environment is a simulation of a soccer field and the unevenness of the field can change the direction of the ball. Therefore, the relationship between "hold the ball time" $T_{0}$ and the speed of "carrying the ball" needs to be adjusted. Among them, "hold the ball time" is the car to keep the ball within the front of the car. The speed of "carrying the ball" becomes faster, the car will "shot" more times within the specified time, and the grade will be better.

Among them, "hold the ball time" can be set by the sensor and algorithm. When the distance between the "ball" and the car reaches about $1.5 \mathrm{~cm}$, the car starts to contact the ball. At this time, the motor speed is controlled to limit the acceleration of the car so that the ball remains in the front. This is the process of "carrying the ball". At the end of this process, the ball can to be released by reducing speed or changing the direction of the car.

The test environment is set as follow: the distance is half of a field, that is, 2 meters. The time when the car moves this distance is set as standard time. Only adjust the "hold the ball time" $T_{0}$ in the algorithm and see its influence on time $t$ in which the car "carrying the ball" through the distance of half field. According to the results, after the time $t$ is normalized, the curve is shown in Figure 9. It can be seen from Figure 9 that when the time $T_{0}$ is $0.6 \mathrm{~s}$, the rate of time of moving through half of the field and standard time is the minimum, so the "ball time" is set to be about $0.6 \mathrm{~s}$.

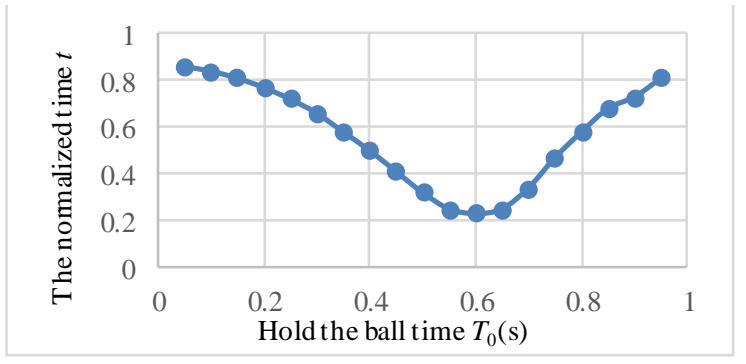

\section{FIGURE VII. INFLUENCE OF “HOLD THE BALL TIME” ON TIME OF MOVING THROUGH HALF OF THE FIELD.}

The second problem encountered during testing is the appropriate radius of the car's turning at obstacles or boundaries. In practice, the car needs to keep the ball in front of the car during the process of "carrying the ball". When the turning radius is set and the turning speed is too large, the ball will run out. The turning speed cannot be too small, otherwise, it will reduce the efficiency of car movement. In order to solve the problem, following experiments are carried out: The turning radius is set between $5 \sim 15 \mathrm{~cm}$, and the max turning speed in each situation is measured. According to Figure 10, as the radius of the obstacle is about $9 \mathrm{~cm}$, while the speed of the car is $6.6 \mathrm{~cm} / \mathrm{s}$. Therefore, the turning radius is set to $10 \mathrm{~cm}$, and the turning speed is set to $6 \mathrm{~cm} / \mathrm{s}$. After two problems are solved, the intelligent car has better performance in straight-ahead movement and corner-avoidance. 


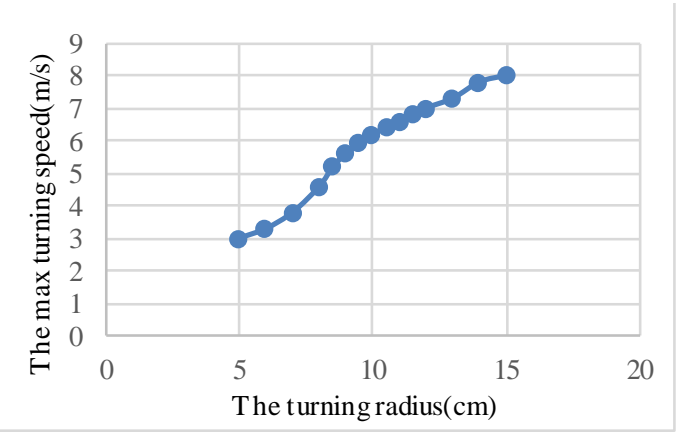

FIGURE VIII. INFLUENCE OF TURNING RADIUS ON THE MAX TURNING SPEED.

\section{CONCLUSIONS}

In this paper, a 3-wheel intelligent car with integrity constraints that can achieve obstacle avoidance and tracking was designed. The kinematics model of the car was deduced, that is, the relationship between wheels speed and the actual speed of the car. And, controlling of the wheel speed was achieved by PWM control method of DC motor. Besides, in order to improve the movement function of the intelligent car, a new PID control algorithm was designed. The parameters of it are adjusted through debugging, such as the "hold the ball time", turning radius and turning speed of the car. Finally, the car got a better movement function and also won a good grade in the soccer racing.

\section{REFERENCES}

[1] XH. Zhao, WG. Qian. "Design of wireless smart car based on single chip microcomputer". Journal of changshu institute of technology, 2016, vol.30, no.4 pp.93-96.

[2] DP. Ma, Y. Li, QO. Liang. "Motion simulation and track drawing of wireless remote control smart car". Journal of zhejiang normal university, 2015, vol.38, no.1 pp.116-120.

[3] Y. Zhen, HM. Zhao, ZL. Peng. "The design of multi-function smart car based on MSP 430". Journal of electronic measurement technology, 2017, vol.40, no.6, pp.10-14.

[4] XJ. Xiang, RJ. Zhou, CZ. Wang. "Design of intelligent detection car control system based on ST M32". Journal of electronic measurement technology, 2016, vol.33, no.4, pp.86-89.

[5] Shafii N, Abdolmaleki A, Ferreira R, et al. Omnidirectional Walking and Active Balance for Soccer Humanoid Robot [C]. Progress in Artificial Intelligence. 16th Portuguese Conference on Artificial Intelligence, EPIA 2013, 283-94.

[6] Gao ZC.Omnidirectional motion control for the humanoid soccer robot[J]. Communications in Computer and Information Science, 2009, vol.51, pp.1-8.

[7] Tsai CC. Kinematics control of an omnidirectional mobile robot[C]. Proceedings of 2005 CACS Automatic Control Conference Tainan, Taiwan, Nov 18-19, 2005, pp.1-6.

[8] Li X. Motion control of an omnidirectional mobile robot[J]. Informatics in Control, Aut omation and Robotics, 2009, vol.3, no.6, pp. 181-193.

[9] ZA. Wang, JJ. Liu. Power Electronics Technique. 5rd ed. China Machine Press, 2005.

[10] Babarsad OB. Modeling and simulating of omni directional soccer $\operatorname{robot}[\mathrm{J}]$. International Journal of Modeling and Optimization, 2012, vol.2, no.5, pp.629-633.

[11] F.Xiang. "Research on intelligent vehicle control algorithm based on indirect PID". Journal of Hubei radio and television university, 2008, vol.28, no.5, pp:155-156. 